\title{
Communications cellulaires, 1975-2006
}

Jean-Pierre Changeux

\section{OpenEdition \\ Journals}

Édition électronique

URL : https://journals.openedition.org/annuaire-cdf/1066

DOI : 10.4000/annuaire-cdf.1066

ISBN : 978-2-7226-0325-7

ISSN : 2109-9227

Éditeur

Collège de France

Édition imprimée

Date de publication : 1 avril 2013

Pagination : 772-776

ISBN : 978-2-7226-0198-7

ISSN : 0069-5580

Référence électronique

Jean-Pierre Changeux, «Communications cellulaires, 1975-2006 », L'annuaire du Collège de France [En

ligne], 112 | 2013, mis en ligne le 22 novembre 2013, consulté le 22 août 2022. URL : http://

journals.openedition.org/annuaire-cdf/1066 ; DOI : https://doi.org/10.4000/annuaire-cdf.1066

Tous droits réservés 


\title{
M. Jean-Pierre CHANGEUX, membre de l'Institut (Académie des sciences)
}

\section{Communications cellulaires, 1975-2006}

\author{
RECHERCHE \\ Un modèle neuronal de codage prédictif rendant compte \\ de la négativité de discordance ou « mismatch negativity »
}

Wacongne C., Changeux J.-P., Dehaene S., «A neuronal model of predictive coding accounting for the mismatch negativity » J. Neurosci, 32(11), 2012, 36653678 [doi: 10.1523/JNEUROSCI.5003-11.2012]

La négativité de discordance ou mismatch negativity (MMN) est considérée comme un indicateur de l'activation de réseaux de neurones spécialisés dans la prédiction active et la détection de déviance. Toutefois, un modèle neuronal détaillé des mécanismes neurobiologiques qui rende compte de la MMN manque et ses fondements computationnels sont débattus. Nous proposons un modèle détaillé du cortex auditif, basé sur le codage prédictif, qui rend compte des aspects critiques de la MMN. Le modèle est intégralement composé de neurones actifs excitateurs et inhibiteurs interconnectés dans une architecture corticale en couche avec des unités d'entrée distinctes, prédictives, et des unités de prédiction d'erreur. Une règle d'apprentissage qui dépend du déroulement temporel des influx nerveux et d'une transmission synaptique impliquant le récepteur NMDA, permet au réseau d'ajuster ses prédictions internes et d'utiliser une mémoire des signaux passés récents pour anticiper les transitions statistiques des stimuli. Nous démontrons que cette architecture très simple rend compte des principales propriétés empiriques de la MMN. Celles-ci incluent une réponse fréquence-dépendante aux déviants rares, une réponse aux répétitions inattendues dans des séquences alternantes (ABABAA...), une absence de considération du contexte global de la séquence, une réponse à l'omission d'un son, et une sensibilité de la MMN aux antagonistes du récepteur NMDA. De nouvelles prédictions sont proposées, et une nouvelle expérience de magnétoencéphalographie chez des sujets humains sains qui valide notre hypothèse de base est présentée. En conclusion, la MMN résulterait d'une prédiction corticale active plutôt que d'une habituation synaptique passive.

\section{La co-activation des neurones dopaminergiques et GABAergiques du VTA interviennent dans le renforcement à la nicotine}

Tolu S., Eddine R., Marti F., David V., Graupner M., Pons S., Baudonnat M., Husson M., Besson M., Reperant C., Zemdegs J., Pages C., Hay Y.A., Lambolez B., Caboche J., Gutkin B., Gardier A.M., Changeux J.-P., Faure P. et Maskos U., "Co-activation of VTA DA and GABA neurons mediates nicotine reinforcement ", Molecular psychiatry, 2012, 1-12 [doi: 10.1038/mp.2012.83]

Le tabagisme est la plus importante cause évitable de mortalité et de maladies à travers le monde. La dépendance à la nicotine fait intervenir le récepteur nicotinique de l'acetylcholine (nRACh), exprimé dans la plupart des neurones et également 
dans plusieurs autres organes du corps. Même dans l'aire tegmentale ventrale (VTA), le territoire critique du cerveau responsable des propriétés « renforçantes » de toutes les drogues addictives, la nicotine agit sur plusieurs types cellulaires et leurs afférences. L'identification de l'effet précis de la nicotine sur ce microcircuit, in vivo, est importante pour comprendre ce renforcement et permettre finalement le développement des traitements efficaces du tabagisme. Nous avons utilisé un nouveau système lentiviral pour ré-exprimer exclusivement le nRACh de hauteaffinité : soit dans les neurones dopaminergiques (DAergique), soit dans les neurones libérant l'acide $\gamma$-aminobutyrique (GABAergique), soit dans les deux au niveau du VTA. Utilisant l'électrophysiologie in vivo, nous montrons que, à la différence des modèles couramment acceptés, l'activation des neurones GABAergiques du VTA joue un rôle crucial dans le contrôle de l'activité DAergique provoquée par la nicotine. Nos résultats démontrent que les valeurs motivationnelles positive et négative sont transmises par le neurone dopaminergique (DA), mais que l'activité concertée des systèmes DA and GABA est nécessaire pour les actions renforçantes de la nicotine en produisant la décharge en rafale des neurones DA. Ce travail identifie les inter-neurones GABAergiques comme cible potentielle des agents anti tabagisme à développer dans l'avenir.

\section{Décider entre motivations en conflit : rôle joué par le cortex préfrontal chez la souris}

Granon S., Changeux J.-P., «Deciding between conflicting motivations: what mice make of their prefrontal cortex », Behavioural Brain Research, 229, 2012, 419-426; epub 18 novembre 2011.

L'étude a porté sur la contribution du cortex préfrontal chez la souris, et plus spécifiquement l'aire pré-limbique, dans une tâche d'apprentissage qui engage un choix entre deux motivations en conflit, l'une pour la recherche de nourriture, l'autre pour la recherche de nouveauté. Nous avons utilisé comme paradigme d'apprentissage une tâche utilisant un labyrinthe en croix qui cible, d'abord, la motivation de conflit et, en second, une règle flexible d'adaptation à un changement d'environnement. Nous montrons que les souris avec des lésions pré-limbiques, qui présentent un apprentissage normal, montrent un déficit lors du passage d'un type de récompense - prise de nourriture - à un autre - recherche de nouveauté - et des difficultés soutenues à adapter leur comportement quand la règle change de manière répétée. Chez la souris, comme chez d'autres mammifères, le cortex préfrontal participe au contrôle de choix flexibles entre motivations naturelles concurrentes et d'adaptation rapide et flexible à des changements externes. Ces résultats ouvrent la voie à des études de modèles de motivation conflictuelle et d'adaptation cognitive, fonctions cérébrales engagées dans plusieurs conditions psychiatriques chez l'homme qui engagent des altérations du cortex préfrontal.

\section{PUBLICATIONS 2011-2012}

Tolu S., Eddine R., Marti F., David V., Graupner M., Pons S., Baudonnat M., Husson M., Besson M., Reperant C., Zemdegs J., Pages C., Hay YAH, Lambolez B., Caboche J., Gutkin B., Gardier A.M., Changeux J.-P., Faure P. et Maskos U., « Co-activation of VTA DA 
and GABA neurons mediates nicotine reinforcement », Molecular Psychiatry, 2012, 1-12 [doi: 10.1038/mp.2012.83].

Wacongne C., Changeux J.-P., Dehaene S., «A neuronal model of predictive coding accounting for the mismatch negativity », J. Neurosci., 32, 2012, 3665-3678 [doi: 10.1523/ JNEUROSCI.5003-11.2012].

Granon S, Changeux J.-P., « Deciding between conflicting motivations: what mice make of their prefrontal cortex », Behav. Brain Res., 229, 2012, 419-426.

Besson M., David V., Baudonnat M., Cazala P., Guilloux J.P., Reperant C., CloezTayarani I., Changeux J.-P., Gardier A.M., Granon S., « Alpha7-nicotinic receptors modulate nicotine-induced reinforcement and extracellular dopamine outflow in the mesolimbic system in mice », Psychopharmacology, 220, 2012, 1-14.

Bourgeois J.P., Meas-Yeadid V., Lesourd A.M., Faure P., Pons S., Maskos U., Changeux J.-P., Olivo-Marin J.C., Granon S., « Modulation of the mouse prefrontal cortex activation by neuronal nicotinic receptors during novelty exploration but not by exploration of a familiar environment », Cereb. Cortex, 22, 2012, 1007-1015.

\section{Revues}

Changeux J.-P., "Allostery and the Monod-Wyman-Changeux model after 50 years », Annu Rev. Biophys., 41, 2012, 103-133.

Changeux J.-P., Edelstein S. « Conformational selection or induced fit? 50 years of debate resolved », F1000 Bio.l Rep., 3, 2011, 19.

Corringer P.J., Poitevin F., Prevost M.S., Sauguet L., Delarue M., Changeux J.-P., «Structure and pharmacology of pentameric receptor channels: from bacteria to brain », Structure, 20, 2012, 941-956.

Changeux J.-P., «Conscious processing: implications for general anesthesia », Curr Opin Anaesthesiol., 25, 397-404; epub 13 juin 2012.

Changeux J.-P., Lou H.C., «Emergent pharmacology of conscious experience: new perspectives in substance addiction », FASEB J., 25, 2011, 2098-2108.

\section{DISTINCTIONS}

The Heller lecture in computational neuroscience, Hebrew University of Jerusalem, 2012.

\section{PRINCIPALES CONFÉRENCES}

\section{Présentation sur invitation dans un cadre national}

Audition: "Processus élémentaires de chimie du cerveau, de plasticité cérébrale et d'apprentissage », organisée par le comité «Mon cerveau face à l'Écran », Académie des sciences, Paris, 14 septembre 2011.

Conférence : « Pour une neuroscience de la personne humaine », organisée Pr B. Ramanantsoa, HEC, Paris, 12 septembre 2011.

Séminaire: «Cerveau et cognition», organisée par l'École polytechnique, Palaiseau, 29 septembre 2011.

Séminaire: «Les récepteurs de neurotransmetteur» série «Imagerie du Vivant», Université Paris Descartes, Paris, 7 octobre 2011. 
Colloque de rentrée du Collège de France: «La vie des formes » organisé par J.-P. Changeux, «Introduction », Paris, 13-14 octobre 2011.

Colloque : «Connoisseurship», organisé par l'École du Louvre: «Physiologie autobiographique du collectionneur », Paris, 20-22 octobre 2011.

Audition avec M.M. Claeys et Vialatte, députés rapporteurs de l'Office parlementaire d'évaluation des choix scientifiques et technologiques, sur «Les enjeux des nouvelles technologies d'exploration et de thérapies du cerveau », Assemblée Nationale, Paris, 15 novembre 2011.

Conférence organisé par Jacques Perié, GREP, Toulouse : «Experimental and Theorical Approaches to Conscious Processing », Toulouse, 6-7 avril 2012.

Audition sur France Inter : «La tête au carré », organisée par Anne-Cécile Perrin, France Inter, Paris, 5 avril 2012.

Conférence à l'École nationale d'administration de Strasbourg : «Pour une neuroscience de la personne humaine », 25 avril 2012.

Conférence : «Acteurs privés du patrimoine » à l'école Condé à Paris, sur : «Physiologie cérébrale, perception de la lumière et des couleurs, perception esthétique » Paris, 11 mai 2012.

Cycle de conférences sur «les primes socialisations» à l'École normale supérieure de Lyon: «Pour une neuroscience de la personne humaine », Lyon, 6 juin 2012

Conférence: «Encuentros à Paris 2012 » à l'Université de Sorbonne, «Pour une neuroscience de la personne humaine », Paris, 5-6 juillet 2012.

\section{Présentations sur invitation dans un cadre international}

Conférence : «La Conscience de l'Art» à l'occasion de la présentation de la thèse de Mr Jan De Maere, «Neuroscience et connoisseurship » organisée par l'Universiteit Gent, Gent, Belgique, 30 septembre 2011.

Conférence plénière «Ethique et neuroscience » à la 41è réunion du Comité Directeur pour la bioéthique (CDBI), organisée par Health and Bioethics Department, Conseil de l'Europe, Strasbourg, 2 novembre 2011.

Conférence plénière \& research conference : «Frontiers in Neuroscience : From Brain to Mind », organisée par le Pr Hitoshi Sakano, de l'IIAS à l'Université de Tokyo et à Kyoto, Japon, 3-10 décembre 2011.

ESF strategic meeting «The Human Brain: from Cells to Society», organisé par Eva Hoagland : conférence «The design of drugs targeted to the brain », à Berlin, Allemagne, 14-15 décembre 2011.

Séminaire «Allostery, Signal Transduction and Drug Design », organisé par Pr Edward Dennis à Division of Biological Sciences, Pharmacology Department and Drug Discovery Institute, Université de Californie, San Diego, 22 février 2012.

Conférence plénière au séminaire: «Franco-Scottish science: systems and molecular Neuroscience », organisé par la Royal Society of Edinburgh : «Experimental and theoretical approaches to conscious processing », à Edinburgh, Scotland, 21-23 mars 2012.

Conférence plénière: «Conscious experience and the molecular chemistry of general anesthesia », Symposium en l'honneur du Pr Hugo Lagercrantz: « Newborn breathing and brain», organisé par Pr Michael Norman, Karolinska Institute \& University Hospital, Stockholm, Suède, 17 avril 2012.

Conférence « Allostery, Signal Transduction and Drug Design », invité par Pr Sten Grillner, Physiological Society of Sweden, Karolinska Institute Stockholm, Suède 19 avril 2012.

Conférence Heller à l'ICNC «Experimental and theoretical approaches to conscious processing », à Safra Brain Center Jerusalem, Israël, 1 mai 2012. 
Memorial Symposium for Vivian Teichberg : «Allosteric properties of ligand-gated ion channels : lessons from prokaryotic receptors », Weizmann Institute Rehovot, Israël, 2 mai 2012.

Conférence : «Genetic and epigenetic constraints on cognitive development », colloque «Variance \& Invariants in Brain Behavior», organisé par Technion Institute of Technology à Haifa, Israël, 21-23 mai 2012.

Conférence : «Genetic and epigenetic constraints on cognitive development », workshop : « Core Knowledge, language and Culture », à Lorentz Center, Leiden, Pays-Bas, du 28 mai au 3 juin 2012.

Conférence : «The Pharmaceutical Colloquium lecture series », à l'Université de Bonn : «Allosteric mechanisms of signal transduction in neurotransmitter receptors : implications for drug design », à Bonn, Allemagne, 2 juillet 2012.

\section{Claude COHEN-TANNOUDJI, membre de l'Institut (Académie des sciences)}

\section{Physique atomique et moléculaire, 1973-2004}

\section{PuBlications}

Wolf P., Blanchet L., Bordé C.J., Reynaud S., Salomon C. et Cohen-Tannoudji C., « Reply to the comment on 'Does an atom interferometer test the gravitational redshift at the Compton frequency?' », Class. Quantum Grav., 29, 2012, 048002.

Wassen W., Cohen-Tannoudji C., Leduc M., Boiron D., Westbrook C.I., Truscott A., Baldwin K., Birkl G., Cancio P., Trippenbach M., «Cold and trapped metastable noble gases », Rev. Mod. Phys., 84, 2012, 175.

\section{Ouvrages}

Cohen-Tannoudji C. et Guéry-Odelin D., «Advances in Atomic Physics - An overview », World Scientific, Singapour, 2011, ouvrage de 750 p.

\section{CONFÉRENCES}

(Invitations à des conférences internationales, conférences spéciales et commémoratives, conférences grand public)

Science and Innovation Week (SIW 2011), série de trois conférences, Mexique, 17, 19 et 21 octobre 2011: «Advances in Atomic Physics. From Optical Pumping to Ultracold Atoms », «Laser Manipulation of Atoms. From Basic Research to Applications ».

Congrès annuel de l'Union des professeurs de physique et de chimie 2011, Université de Montpellier 2, 24 octobre 2011: «Atomes et photons : de la recherche fondamentale aux applications ». 